\title{
PULMONARY EMBOLISM: THE CLINICAL AND CARDIOGRAPHIC PROGRESS OF A CASE BY
}

\author{
DAVID LEWES
}

From the Southend General Hospital, Southend

Received July 21, 1944

Although severe pulmonary embolism is not an uncommon complication of abdominal operations, the opportunities for a close clinical study of the condition are few. Too often the medical officer arrives at the bedside when it is too late. Even those patients who do not succumb at once rarely survive a few hours. In such circumstances, emergency measures connected with treatment take the place of clinical observations, which might include cardiographic records.

The account that follows is about a patient who developed severe pulmonary embolism, eight days after abdominal hysterectomy, and was observed continuously from the onset of the embolism until recovery took place.

\section{CASE Notes}

A woman, aged 42, was admitted to hospital for the investigation of ascites, which had lately set in with shortness of breath and anorexia. A hard and slightly tender nodular mass, rising out of the pelvis to the left of the midline, was considered to be an ovarian cyst and probably malignant. At laparotomy, free fluid was found in the peritoneal cavity; it had arisen in connection with papilliferous ovarian cysts, one of which had ruptured. The uterus, densely adherent to the bladder and rectum, was successfully removed with both cysts, which proved to be carcinomatous on microscopic examination.

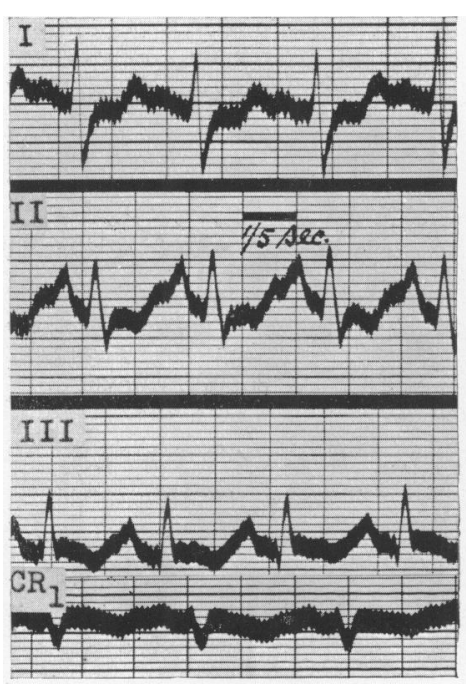

A

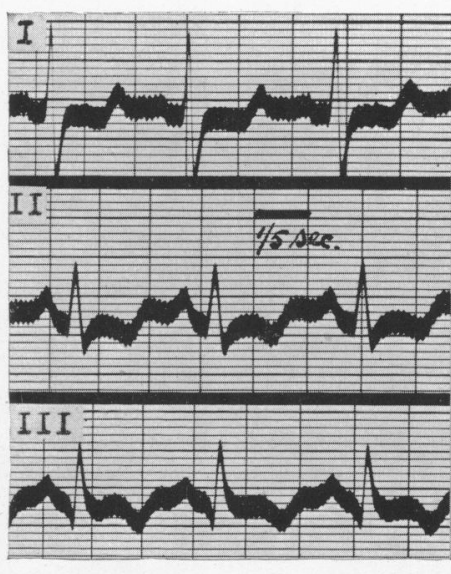

B

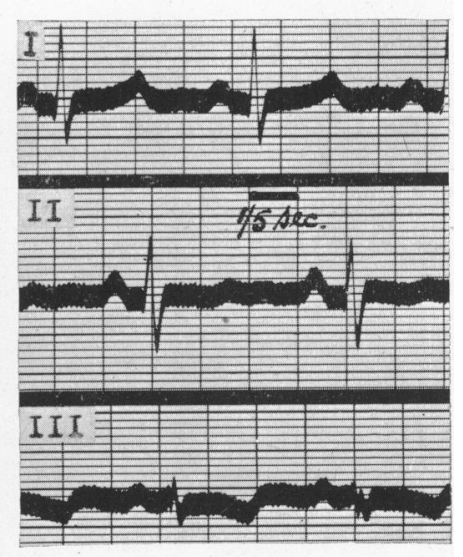

C

FIG. 1.-Serial cardiograms after pulmonary embolism (20/5/43), with inversion of $\mathrm{T}$ in leads II, III, and $\mathrm{CR}_{1}$ (see p. 163).
(A) $20 / 5 / 43$, the same evening.
(B) $21 / 5 / 43$, the next day.
(C) $25 / 5 / 43$. 


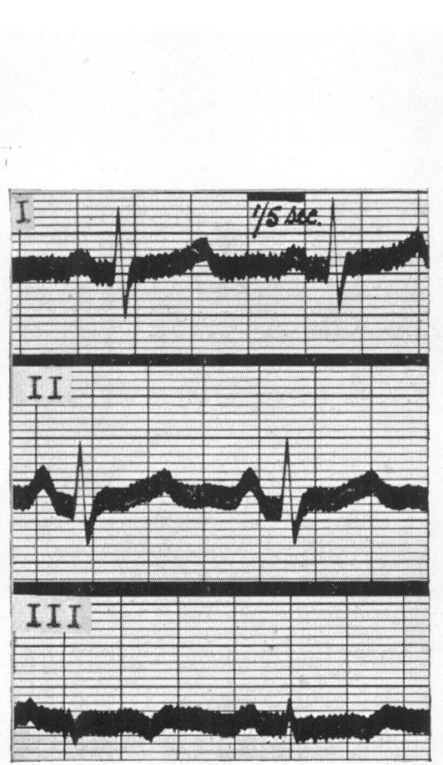

A
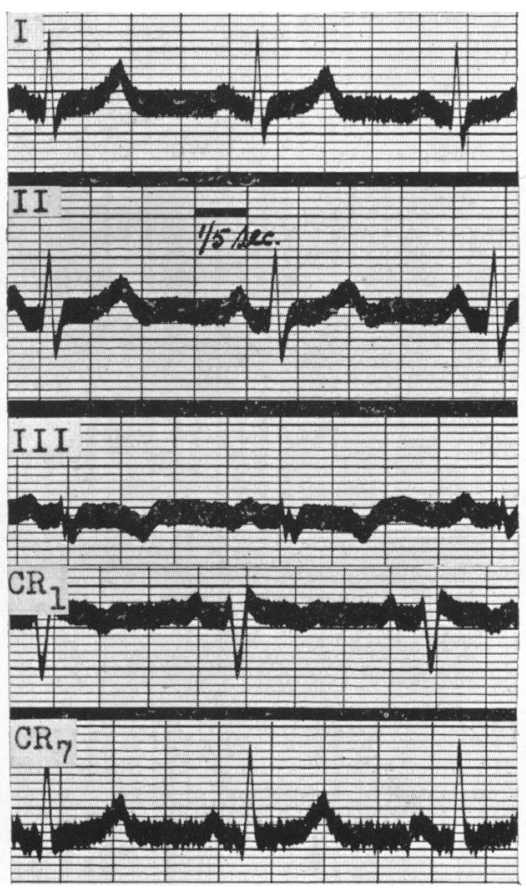

B

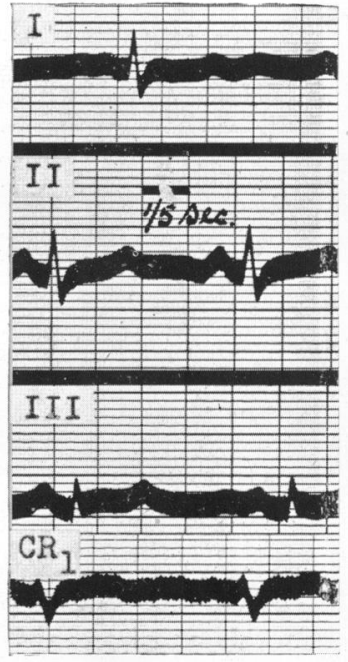

C

Fig. 2.-Subsequent cardiograms showing recovery.
(A) $27 / 5 / 43$, and
(B) $1 / 6 / 43$, with inverted T III.
(C) $9 / 7 / 43$, with upright T III.

On the eighth day after operation, the patient had a feeling of tightness across the chest and some breathlessness. The symptoms increased during the afternoon, and that evening the discomfort had given way to a feeling of suffocation. She called a nurse who noticed that the breathing had become distressed and that the pulse could not be felt. Five minutes later the patient became suddenly very dyspnœic and turned ashen grey. Gasping violently for breath, she called out "Give me air or I will die." Within thirty seconds she lost consciousness and became pulseless. The pupils dilated widely and the corneal reflex disappeared. There was spontaneous evacuation of urine and fæces.

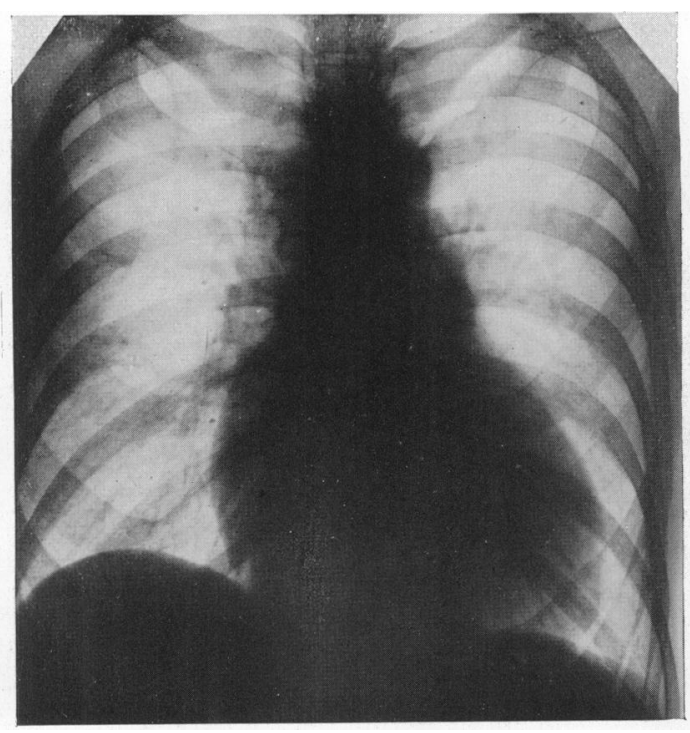

A

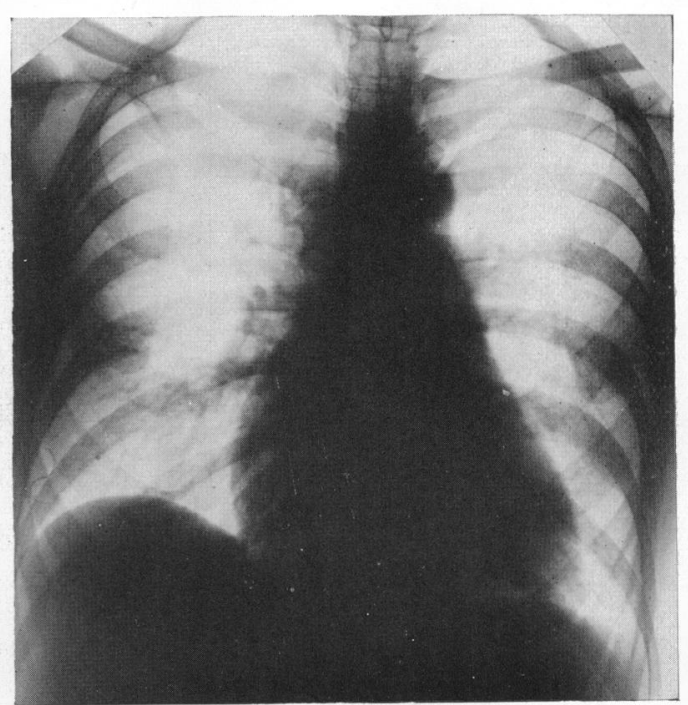

B

Fig. 3.-Teleradiograms showing cardiac enlargement, especially of the right side, and subsequent improvement.
(A) $21 / 5 / 43$, fourteen hours after pulmonary embolism.
(B) $27 / 5 / 43$. 
On auscultation of the heart, an obvious triple rhythm was heard, internal to the apex. Accurate placing of the additional heart sound was impossible at the time, owing to the severe tachycardia (140 a minute). The respiration, at first deep and rapid, became shallow and more rapid (40 a minute) during the period of unconsciousness, which lasted three minutes. The patient suddenly regained full consciousness, sweated profusely, and spoke in a whisper to those at the bedside. The pulse returned. In half an hour the respiration rate had returned to 18 a minute and distress was absent, although exhaustion was still present. The pulse continued to be small and rapid (132 a minute) and the blood pressure was $90 / 80$. The apex beat could not be felt. The heart sounds were distant and there was accentuation of the pulmonary second sound. The triple rhythm persisted.

Next dny the patient's condition deteriorated rapidly; the pulse rate dropped from 120 to 76, and the respirations became slow and deep ; but these changes did not last and improvement again took place. With the slower heart rate it was possible to tell that the triple rhythm was caused by the addition of the third heart sound. The blood pressure also improved to 115/75. Harsh breath sounds were heard over the midzone of the left lung but no dullness or moist sounds developed. There was great improvement in the patient's condition on the third day and her recovery appeared to be assured. On the fourth day after pulmonary embolism triple rhythm gave way to dual rhythm, and the blood pressure rose to 130/85. The patient remained apyrexial throughout the illness. There was at no time any complaint of cough or sharp pain in the chest, and hæmoptysis was absent. Oxygen was administered from the onset of the illness and was continued for 25 hours. In the hope of preventing the spread of thrombosis 20,000 units of heparin were administered intravenously on the first day, and 5,000 units two hourly for four doses when di-coumarin (100 mg. by mouth daily for 14 days) was substituted. With the object of decreasing arterial spasm at the site of the embolus, 1 c.c. of eupaverin was given intramuscularly 4-hourly for four doses. Frequent cardiograms were recorded throughout the illness. They confirmed the diagnosis of sudden failure of the right heart and served as an index of recovery of the heart. The serial tracings (Fig. 1 and 2) showed right ventricular preponderance and inversion of the T waves in leads II, III, and in $C_{1}$; the $T$ wave in $C R_{7}$ was upright. Serial teleiadiograms (Fig. 3) were also taken and these showed alteration in the size of the heart contingent with the severity and duration of the obstruction in the pulmonary circulation. They failed to reveal any evidence of pulmonary infarction.

\section{Discussion}

The observations of McGinn and White (1935) that consistent variations from the normal appeared in the cardiograms of a number of their patients developing pulmonary embolism have given impetus to the study of the behaviour of the heart in this condition. Their results have been repeatedly confirmed and extended by numerous workers, particularly by Barnes (1936), who tabulated in detail the diagnostic features of the limb lead changes occurring in pulmonary embolism, and by Wood (1941), who directed attention to the significance and importance of multiple chest leads in the differential diagnosis of acute cor pulmonale. In spite of these notable advances, as Murnaghan and others (1943) have pointed out in a review of 102 cases of pulmonary embolism, confusion is liable to arise in an interpretation of the cardiographic changes, unless a distinction is made between cases with and without acute cor pulmonale and cases with and without pre-existing heart disease. Furthermore it is only by assigning a case of pulmonary embolism to its appropriate category that a correct interpretation of the clinical and cardiographic findings is possible. The case reported, having developed a severe degree of acute cor pulmonale in the absence of pre-existing heart disease, affords a good opportunity for undisturbed observation of the effects on the heart of pulmonary embolism, and has allowed its close study which has already extended over twelve months. In addition, certain clinical observations, dating from the moment of embolism, early cardiographic tracings and serial radiograms of the heart have yielded information in regard to the mechanism of acute cor pulmonale.

Pulmonary obstruction compatible with survival. Experimental evidence would indicate that pulmonary obstruction may be considerable without extinguishing life. Thus, Haggart and Walker (1923) by quantitative closure of the pulmonary artery in cats found that, when up to 52 per cent of the lumen was occluded, few ill-effects were observed, but that when occlusion exceeded 66 per cent the animals invariably died. Clamping the left pulmonary artery raised the pulmonary arterial pressure by 29 per cent and the respiratory rate by 25 per cent without 
altering the size of the heart, the cardiac output, or the systemic arterial blood pressure. From the clinical aspect, the findings of Thompson and Evans (1930) show that the upper limit of acute depletion of the pulmonary circulation compatible with survival, is under 50 per cent. Studying the problem of paradoxical embolism and its relation to pulmonary embolism, they concluded that if 50 per cent or more of the pulmonary circulation be cut off suddenly by an embolus, death results within 10 to 30 minutes. Thus in 18 cases of pulmonary embolism, in which embolus was the cause of death proved at autopsy, 16 showed depletion of the pulmonary circulation by 50 per cent or more ; only one of these 16 cases had survived a period of 30 minutes.

Severe pulmonary obstruction without infarction. The clinical, radiological, and cardiographic evidence in the case reported, indicates severe initial pulmonary obstruction which became steadily regressive and finally ceased. The most likely explanation for this behaviour -applicable to other cases of acute cor pulmonale which survive-is that much of the severe but temporary obstruction is caused by vasospasm throughout the pulmonary tree as a result of the embolism (Jesser and de Takáts, 1941). This explanation is rendered all the more certain in the present case, in that there was neither clinical nor radiological evidence of pulmonary infarction. Such negative evidence would also indicate that the embolus initiating pulmonary obstruction was of small size. It is not easy to envisage an embolus that could bring about a severe degree of obstruction by virtue of size alone being driven into the pulmonary tree and yet failing to produce an infarct. But even allowing that the embolus were small, why did it not declare itself by signs of pulmonary infarction ? The only explanation accounting for such a contingency is found in the unusually rich anastomosis existing between the bronchial and pulmonary arteries, preventing pulmonary infarction. Anastomoses of this kind, an exaggeration of the normal, are seen for example in a gross and chronic form in congenital pulmonary atresia.

The clinical diagnosis of acute cor pulmonale. McGinn and White (1935) described acute cor pulmonale as the result of sudden distension of the right ventricle and right auricle which might follow obstruction to the pulmonary artery by an embolus. The condition can freequently be recognized by a knowledge of the previous history and by attention to the early symptoms and signs as typified by the present case. Severe initial shock, pallor, cold extremities, great dyspnœa, and low blood pressure are important signs in the clinical diagnosis of acute cor pulmonale. Thus Murnaghan and others (1943) in two series of cases of pulmonary embolism, presenting with and without signs of shock, found cardiographic evidence of acute cor pulmonale in just over half of the first group and in under a quarter of the second. Distension of cervical veins, although not recorded in the present case, is claimed by Wood (1941) to be present in all cases of acute cor pulmonale in which this sign is sought within the first three days of embolism. After this period venous distension is often absent.

A physical sign of great diagnostic value is the early appearance of triple rhythm due to the third heart sound. Within a few minutes of embolism in the present case a distinct triple rhythm was heard, and although its exact nature during tachycardia was difficult to interpret it became obvious when the beat slowed. Triple rhythm in acute cor pulmonale has been recorded by several observers (Murnaghan et at., 1943 ; Wood, 1941), but without emphasis on its value in clinical diagnosis. The addition of the third heart sound, when not a physiological finding in young adults, is a reliable clinical index of right-sided heart failure (Evans, 1943). Thus it was commonly heard in mitral stenosis, hypertensive heart failure, emphysema, and congenital heart disease.

The early appearance of the third heart sound in the present case was clinical proof of acute cor pulmonale because of the known absence of previous heart disease. This sign should rank high amongst the clinical criteria by which the diagnosis of acute cor pulmonale is determined. Less severe pulmonary embolism producing lesser degrees of acute cor pulmonale may not show triple rhythm, and recourse to electrocardiographic records may be necessary 
to supply evidence of right ventricular stress, but it suggested that every case of pulmonary embolism presenting triple rhythm (Type Ib of Evans) demonstrates acute cor pulmonale as well, provided that pre-existing heart disease can be excluded.

Changes in the size of the heart. Cardiac enlargement, predominantly of the right side, shown radiologically 14 hours after embolism (Fig. 3), would indicate that actual distension of the right ventricle and right auricle was an important factor in determining the striking initial cardiographic changes, which persisted in a modified and steadily regressive form for a period of seven weeks. Besides demonstrating that acute ventricular distension, compatible with survival, can take place in the absence of radiographic evidence of pulmonary infarction, the X-ray appearance of the heart is in keeping with the post-mortem findings of right ventricular and right auricular enlargement evidenced in some fatal cases of pulmonary embolism.

Electrocardiographic changes. Cardiographic irregularities in pulmonary embolism are characteristic, and when present they confirm the presence of acute cor pulmonale. Briefly they are, prominent S I with the S-T segment starting slightly below the base line ; depressed RS-T segment in lead II, with gradual ascent from the $S$ to the $T$ wave in the same lead ; usually a diphasic or monophasic $\mathrm{T} \mathrm{II}$; the presence of a $\mathrm{Q}$ wave and definite inversion of $\mathrm{T}$ in lead III (McGinn and White, 1935); sharp inversion of T without appreciable displacement of the RS-T segment, always in $\mathrm{CR}_{\mathbf{1}}$, and sometimes for shorter duration in IV R ; $T$ in $\mathrm{CR}_{\mathbf{1}}$ commonly remains inverted for several weeks ; less essential is a tendency for the QRS deflection to be mainly upward in $\mathrm{CR}_{1}$ (Wood 1941).

My case showed such changes, some of them strikingly. Right axis deviation was noteworthy, with an unusually deep S I and sharp inversion of $T$ in leads II, III, and $\mathbf{C R}_{1}$. Thus the cardiographic records confirmed the conclusions reached in regard to the early appearance of the third heart sound and the radiographic evidence of right ventricular distension. The chest leads show that recovery was delayed longest in $\mathrm{CR}_{1}$, and that even after seven weeks, the $T$ wave although upright was low. Thus, $\mathbf{T}$ in $\mathbf{C R}_{1}$ is the most sensitive cardiographic index we possess for determining the presence of right ventricular strain. The direction of the $T$ wave in lead $\mathrm{CR}_{7}$ should be noted. Evans and Hunter (1943) in describing this lead implemented it in distinguishing between T II and T III inversion of posterior cardiac infarction and similar changes seen in heart failure in emphysema and in pericardial and congenital heart disease. In these conditions $T$ in $\mathrm{CR}_{7}$ is positive, with recognized exceptions, while the reverse is usually the case in posterior cardiac infarction. In my case, therefore, a positive $\mathrm{T}$ in $\mathrm{CR}_{7}$ demonstrates that their observations apply equally to a condition characterized by acute right ventricular failure as they do to the chronic forms of right sided failure which they described.

Myocardial ischæmia-resulting from coronary insufficiency and variously attributed to increased tension in the right ventricle, shock, asphyxia, fall in systemic arterial pressure, and reflex changes in the coronary circulation-has been put forward to account for the cardiographic changes in pulmonary embolism (Horn et al., 1939). Although cardiac ischæmia undoubtedly takes place in the development of acute cor pulmonale and may even proceed to cardiac infarction, in the absence of coronary occlusion (Currens and Barnes, 1943), it cannot be advanced as the cause of the limb lead changes. Wood (1941) had demonstrated that although the limb lead changes in pulmonary embolism and posterior cardiac infarction may simulate each other, the chest lead changes in the two conditions are entirely different. More recently (Evans and Hunter, 1943) the $\mathrm{CR}_{7}$ chest lead has helped in the differential diagnosis as instanced by my case. Further, the similarity of cardiographic behaviour in pulmonary embolism and in conditions characterized by chronic right ventricular strain, points to acute right ventricular stress as being the cause of the cardiographic changes in pulmonary embolism. Cardiographic changes similar to those seen in pulmonary embolism may be recorded in pulmonary stenosis, mitral stenosis, and chronic cor pulmonale (Selzer and Wood, 1939), while in rheumatic carditis, which may be associated with isolated right ventricular fallure, Wood 
(1941) has recorded transient changes, indistinguishable from those found in pulmonary embolism.

\section{SUMMARY AND CONCLUSIONS}

A case of pulmonary embolism with acute cor pulmonale is described. A severe degree of cor pulmonale developed in the absence of radiological evidence of pulmonary infarction.

Emphasis has been placed on the value of triple rhythm, from the addition of the third heart sound; in the diagnosis of suspected cases of pulmonary embolism.

Radiographic evidence of distension of the right auricle and right ventricle is related to the onset and duration of the cardiographic changes. Inversion of the $\mathrm{T}$ wave in $\mathrm{CR}_{1}$ is probably the most sensitive of the cardiographic indices of right ventricular failure. The value of this change and of findings in lead $\mathrm{CR}_{7}$ in the diagnosis of acute cor pulmonale from posterior cardiac infarction is confirmed. .

I wish to thank Dr. R. Sleigh Johnson, under whose care the patient was admitted to the Southend General Hospital, for his permission to publish this case and for the interest he has shown in this work. Particularly do I wish to thank Dr. William Evans for his constant encouragement and for his helpful criticism of this paper. The keen co-operation of Miss Peach, technician to the Cardiac Department, and of Mr. W. E. Beadle, who took the radiographs, is much appreciated.

\section{REFERENCES}

Barnes, A. F. (1936). Proc. Staff Meet., Mayo Clin., 11, 11.

Currens, J., and Barnes, A. R. (1943). Arch. intern. Med., 71, 325.

Evans, W. (1943). Brit. Heart J., 5, 205.

- and Hunter, A. (1943). Ibid., 5, 73.

Haggart, G. E., and Walker, A. M. (1923). Arch. Surg., 6, 764.

Horn, H., Dack, S., and Friedberg, C. K. (1939). Arch. intern. Med., 64, 296.

Jesser, J. H., and de Takáts, G. (1941). Arch. Surg., 42, 1034.

McGinn, S., and White, P. D. (1935). J. Amer. med. Ass., 104, 1473.

Murnaghan, D., McGinn, S., and White, P. D. (1943). Amer. Heart J., 25, 573.

Selzer, A., and Wood, P. (1939). Brit. Heart J., 1, 49.

Thompson, T., and Evans, W. (1930). Quart. J. Med., 23, 135.

Wood, P. (1941). Brit. Heart J., 3, 21. 